\title{
Clinicopathological and Survival Characteristics of Malignant Pleural Mesothelioma: A Single-Institutional Experience
}

\author{
Şule KARABULUT GÜL, ${ }^{1}$ Ahmet Fatih ORUÇ, ${ }^{1}$ Özlem ORUÇ² \\ 'Department of Radiation Oncology, Dr. Lütfi Kırdar Kartal Training and Research Hospital, Istanbul-Turkey \\ ${ }^{2}$ Department of Chest Diseases, Süreyyapaşa Chest Diseases Training and Research Hospital, Istanbul-Turkey
}

\begin{abstract}
OBJECTIVE
A single-institution study of 7 years of experience was performed to investigate the clinicopathological and therapeutic characteristics of malignant pleural mesothelioma (MPM) patients who received multimodality treatment, as well as factors affecting survival.
\end{abstract}

\section{METHODS}

A retrospective review of a consecutive cohort of pathologically confirmed MPM patients who were referred to our radiation oncology clinic and received multimodality treatment strategy that included surgery, chemotherapy, and radiotherapy at our training and research hospital between 2001 and 2008 was performed.

\section{RESULTS}

A total of 53 patients were included in the study. Median age was 58 years (range: 33-79 years). Mean duration of follow-up was 14 months (range: $4-82$ months). Disease-free status and overall survival were 11 months (range: 3-50 months) and 14 months (range: 4-82 months), respectively. Factors that individually predicted better prognosis were younger age ( $<50$ years), having undergone surgery, having received radiotherapy, and having undergone multimodality treatment.

\section{CONCLUSION}

Further comprehensive and randomized studies are required to better understand biological behavior of MPM in order to obtain more successful results in the management of the disease.

Keywords: Chemotherapy; malignant pleural mesothelioma; multimodality treatment; radiotherapy; survival. Copyright $\odot$ 2017, Turkish Society for Radiation Oncology

\section{Introduction}

Malignant pleural mesothelioma (MPM) is a relatively rare and highly lethal tumor induced by asbestos exposure, with a growing incidence over the last decades. $[1,2]$ As it has a highly aggressive behavior, there have been some studies to identify more accurate prognostic factors and staging systems, and to investigate novel treatment regimens.[1] However, the relative rarity of this neoplasm has limited research opportunities, and only a few clinical trials have been done or are on-going.[2]

In this retrospective study, we reported the single institutional 7 years experience of the clinicopathologic and therapeutic characteristics and the factors affecting survival and the factors that individually predicted better prognosis in patients with MPM. 


\section{Materials and Methods}

\section{Patients and data retrieval}

This was a retrospective review of a consecutive cohort of pathologically confirmed MPM patients who referred to our radiation oncology clinic and treated with multimodality treatment strategy including surgery, chemotherapy and radiotherapy at our Training and Research Hospital between 2001 and 2008.

Clinical records of our Radiation Oncology Clinic registry were analyzed for sex, age, exposure to asbestos, Eastern Cooperative Oncology Group (ECOG) performance status, tumor cell type, lymph node involvement, stage of the disease, treatment modalities, chemo-and radiotherapy-related adverse events, follow-up period, response to treatment, site of metastasis and data on survival.

The patients were staged based on the pathologic and clinical findings, including imaging studies, according to TNM staging system proposed by the International Mesothelioma Interest Group (IMIG) Staging. [3] Imaging studies included thorax CT or MRI and bone scintigraphy or PET-CT, when indicated.

\section{Multimodality management}

A group of patients were referred to our clinic after surgery. Depending on whether the treatment goals were palliative or curative, 4 surgical options were performed for these patients. 11 patients had gone EPP, 6 patients had decortication, pleurodesis was established for 5 of them, and two of them had radical pleuroctomy. Extrapleural pneumonectomy (EPP) was defined as an en bloc resection of the pleura, lung, ipsilateral diaphragm, and pericardium. Radical pleurectomy/ decortication, which removed all gross tumor without removing underlying lung, was performed in patients who had minimal visceral pleural tumor or poor pulmonary function.

The choice of chemotherapy agent, dose, and schedule were at the discretion of the treating medical oncologist. Before chemotherapy administration, following criteria were established; adequate bone marrow function as indicated by: platelets $\geq 100000 / \mathrm{mm}^{3}$ and hemoglobin $10 \mathrm{~g} / \mathrm{dL}$ and neutrophils $>1.5 \times 10^{3} /$ $\mathrm{mm}^{3}$; adequate renal function as indicated by serum creatinine: $<1.5 \mathrm{x}$ the upper limit of normal; adequate liver function as indicated by serum bilirubin level $<1.5$ $\mathrm{x}$ the upper limit of normal and AST or ALT less than two times the upper limit of normal.

First and second line regimens were used in chemotherapy for 46 patients. We used gemcyitabine and cisplatin for 13 of them, pemetrexat and cisplatin for 30 of them, 2 of them had adrioblastyn and one of them had etoposyd.

Gemcitabine was administered $1200 \mathrm{mg} / \mathrm{m}^{2}$ on the $1^{\text {st }}$ and $8^{\text {th }}$ day, cisplatin $75 \mathrm{mg} / \mathrm{m}^{2}$ on the $1^{\text {st }}$ day once in every 21 days, pemetrexed $500 \mathrm{mg} / \mathrm{m}^{2}$ on the $1^{\text {st }}$ day and cisplatin $75 \mathrm{mg} / \mathrm{m}^{2}$ on the first day once in every 21 days.

After chemotherapy, radiotherapy was performed in a group of patients for adjuvant or palliative purposes for 26 patient. So that we didn't have Conformal RT or IMRT devices at the time of the study, we used Conventional RT. Two different doses were applied; $55 \mathrm{~Gy}$ were applied for 9 patients and 46 Gy were applied for 22 patients. The combination of photon and electron was used as a radiotherapy technique. Patients who underwent EPP were given 46 Gy beam in 23 fractions covering whole hemithorax, mediastinum, surgical and drainage scars and subsequently, higher doses were administered, by protecting medulla spinalis and heart.

For cases who underwent partial pleurectomy or who couldn't be operated, a photon- electron combination was preferred. The area of lung was determined in conventional simulator and an appropriate block was poured. 6-15 MV photon beam was given to peripheral areas using linear accelerator (GE Saturn 41) by providing lung protection and subsequently electron energy of 9-12 meV was applied to the covered lung volume from both front and back side.

\section{Response to treatment and survival}

Responses (complete response, partial response, stationary disease and progressive disease) to treatment were defined in accordance with the criteria by World Health Organization (WHO handbook for reporting of cancer treatment. Geneva) and post-treatment response was assessed via thoracic CT or MRI. Time to progression was defined as the time from diagnosis to relapse, metastases or death due to other reasons before development of relapse, whereas overall survival was defined as the time from diagnosis to death.

\section{Statistical methods}

Several factors, including; age, gender, epithelial histology, stage, type of treatment modality, type of surgical intervention, chemotherapy regimen, the dosage of radiotherapy were analyzed to whether they have any influence on survival or not.

Data were entered in data base and statistical tests were performed using SPSS 13. Kaplan-Meier method was used in analysis of survival. $\mathrm{P}$ value was taken as significant if found to be less than 0.05 . 


\section{Results}

A total of 53 patients were identified and included into this retrospective study. 29 of patients were male (54.7\%) and 24 were female (45.3\%). The median age was 58 (33-79). The status of performance on admission was ECOG 0 in four patients (7.5\%), ECOG 1 in 44 patients (83\%) and ECOG 2 in five patients (9.4\%). In etiology, the role of asbestos was observed in $6 \mathrm{pa}-$ tients (11.3\%).

In terms of histological subtypes, 35 patients $(66 \%)$ were epithelial, three (5.7\%) were sarcomatous, seven (13.2\%) were mixed type and eight (15.1\%) had undefined pathology.

In terms of stages, 17 patients (32.1\%) were stage 1 , $18(34 \%)$ were stage $2,7(13.2 \%)$ were stage 3 and 11 (20.8\%) were stage 4.

A total of 19 patients were referred to our clinic after surgery. Twenty- six patients combined treatment with radiotherapy plus chemotherapy and trimodality were performed in 12 patients, respectively. The treatments were in general well tolerated, but the serious adverse events were observed, including grade III-IV myelosuppression [Grading of hematologic toxicity was based on the NCI Common Terminology Criteria for Adverse Events version 3.0. Grade 3 (severe) and grade 4 (life-threatening) hematologic toxicities were noted as follows: hemoglobin (grade $3,<8 \mathrm{~g} / \mathrm{dl}-6.5 \mathrm{~g} /$ $\mathrm{dl}$; and grade $4,<6.5 \mathrm{~g} / \mathrm{dl}$ ); neutrophils (grade $3,<1000 /$ $\mathrm{mm}^{3}-500 / \mathrm{mm}^{3}$ and grade $\left.4,<500 / \mathrm{mm}^{3}\right)$; and platelets (grade 3, $<50,000 / \mathrm{mm}^{3}-25,000 / \mathrm{mm}^{3}$; and grade 4 , $\left.<25,000 / \mathrm{mm}^{3}\right)$ ] and radiation pneumonia determined by physical examination and confirmed radiologically in 4 and 6 patients, respectively.

The mean duration of follow-up was 14 months (4$82)$. The number of patients who responded to treatment was 29 (54.7\%). Post-treatment response was assessed via thoracic CT or thoracic MR and complete response was achieved in two patients (3.8\%) and partial response in 12 patients (22.6\%). 34 patients (64.2\%) had stable disease. Post-treatment progression was detected in 5 patients as locoregional relapse (9.4\%). No abdominal relapse was observed. 17 of patients $(32.1 \%)$ are still alive. Disease-free survival is 11 months (3-50) and overall survival is 14 (4-82) months.

Although, survival according to gender was not detected to be significantly different $(\mathrm{p}=0.079)$, overall survival was established to be longer in women. Overall survival and disease-free survival according to age were found to be higher in patients aged under 50 ( $\mathrm{p}=0.04$ for overall survival, $\mathrm{p}=0.033$ for disease-free survival). Although epithelial histology was superior numerically to histological type in terms of overall survival and disease-free survival in the study, it did not reach statistical significance $(\mathrm{p}=0.682$ for overall survival, $\mathrm{p}=0.617$ for disease-free survival).

Overall survival was found to be statistically significantly higher in the group with surgery when compared to the group without surgery $(\mathrm{p}=0.007)$, while overall survival according to types of surgery was not statistically significantly different ( $\mathrm{p}=0.909)$.

Overall survival was found to be statistically significantly longer in patients who received radiotherapy $(p=0.001)$. Survival was observed statistically significantly higher in the group with both chemotherapy and radiotherapy $(\mathrm{p}=0.001)$.

Most importantly overall survival was found to be statistically higher in the trimodality group $(\mathrm{p}=0.01)$. Six $(66.7 \%)$ of nine patients who were given radiotherapy over 50 Gy are still alive and $18(81.8 \%)$ of 22 patients with lower than 50 Gy are alive. The effect of radiotheraphy dose on overall survival was not statistically significant $(\mathrm{p}=0.677)$.

The remaining parameters failed to be significantly associated with survival in the univariate analysis.

\section{Discussion}

Respiratory exposure to asbestos plays a pivotal role in the etiology MPM.[4] However few cases with tumors originating from tunica vaginalis, testicles and ovarian epithelium have also been described in the literature. [5,6]

Since respiratory exposure to asbestos which plays a pivotal role in the etiology pleura is the most commonly (90\%) encountered location. [4,7,8]

In a number of series published, exposure to asbestos has been reported in $50-80 \%$ of cases with malignant pleural mesothelioma. In our study, six (11.3\%) patients declared exposure to asbestos by anamnesis.

Mesothelioma is a well-known complication of therapeutic radiation for lymphoma, breast cancer, lung cancer, and other malignancies. Patients with Hodgkin lymphoma, for example, experience a 20 -fold increased risk of mesothelioma after radiotherapy.[9] As a matter of fact, we identified the asbestos exposure just in $6(11.3 \%)$ patients.

The mean age of presentation in malignant pleural mesothelioma is 60 years. $[10,11]$ Scagliotti et al. reported that the disease appears frequently in $5^{\text {th }}$ and $6^{\text {th }}$ decades and is more common among men than women (3.6:1).[12] In the present study median age 
was $58(33-79)$ and $29(54.7 \%)$ of patients were men and $24(45.3 \%)$ were female, which is consistent with the literature. In recent two studies. MPM was found to be five times more common in men than women in Britain and mean age of disease onset was 45-85 (72) years. $[13,14]$

Several factors affecting prognosis in malignant pleural mesothelioma include age, gender, serum LDH level, thrombocyte count and performance status. However, a number of studies published have reported that early stage (Stage-I and II), epithelial type, N0 disease and complete resection enhance survival.[15,16]

A study of 183 patients by Sugarbaker et al. concluded that patients with epithelial histology had a superiority over other histological types in terms of overall survival (the mean overall survival is 26 months for epithelial type $(\mathrm{p}=0.001)$.[17]

In a number of studies, mean survival has been reported to be $4-12$ months in patients with nonepithelial histology.[15-19] The present study included 35 patients with epithelial histology and overall survival $(\mathrm{p}=0.682)$ and disease-free survival $(\mathrm{p}=0.617)$ were numerically higher, but not statistically significant, in comparison to patient groups with other histological types.

Our patient's main clinicopathologic characteristics is similar to the other main clinicopathologic characteristics of MPM patients. Epithelial type was the most common tumor histologic type. However, only a small number of patients were given multimodality therapy. By analyzing all, the prognosis of our series was dismal: overall survival is 14 (4-82) months.

Our data reveal the individual effect on survival of the following patient-related survival factors: younger age ( $<50$ years), having undergone surgery, having received radiotherapy, having undergone combined chemotherapy and radiotherapy and finally having undergone trimodality treatment.

Recent studies have focused on the identification and evaluation of potential prognostic factors for survival in MPM patients. According to Borasio et al. significant predictors of survival include performance status, platelet count, histology and degree of involvement of pleural cavity.[1] According to Yan TD et al. asbestos exposure, negative lymph node involvement and receipt of adjuvant radiation were associated with improved survival.[20] Flores et al. reported that standard uptake value greater than 10 , mixed histology and stages III and IV are poor risk factors in malignant pleural mesothelioma.[21]

Treasure T. et al. aimed to assess the clinical out- comes of patients who were randomly assigned to EPP or no EPP in the context of trimodal therapy in the Mesothelioma and Radical Surgery (MARS) feasibility study. 23 patients in the EPP group and 26 in the no EPP group consented to quality-of-life assessment and 12 and 19 patients completed the quality-of-life questionnaires, respectively. Median quality- of-life scores seemed to be lower for the EPP group than the no EPP group, with the lowest median score shortly after surgery; however, there were no statistically significant differences between treatment groups.[22]

A few of the major limitations of our series are the retrospective nature of the study, limited number of patients, particularly in subgroups analysis, and lack of control group; however, we tried to compare our data with available data in the literature.

In conclusion, this retrospective study reflects the single institutional 7 years experience on consecutive cohort of pathologically confirmed MPM patients who referred to our radiation oncology clinic and treated with combined or multimodality treatment strategy including surgery, chemotherapy and radiotherapy. Disease-free survival is 11 months (3-50) and overall survival is $14(4-82)$ months in our series. Factors that individually predicted better prognosis were younger age ( $<50$ years), having undergone surgery, having received radiotherapy, having undergone combined chemotherapy and radiotherapy and finally having undergone trimodality treatment. Further comprehensive and randomized studies are required to understand better biological behaviour of MPM and to obtain more successful results in the management. Hence, currently the most appropriate approach should be a modality treatment in accordance with characteristics of the patient.

\section{Disclosure Statement}

The authors declare no conflicts of interest.

\section{References}

1. Borasio P, Berruti A, Billé A, Lausi P, Levra MG, Giardino R, et al. Malignant pleural mesothelioma: clinicopathologic and survival characteristics in a consecutive series of 394 patients. Eur J Cardiothorac Surg 2008;33(2):307-13.

2. Montanaro F, Rosato R, Gangemi M, Roberti S, Ricceri F, Merler E, et al. Survival of pleural malignant mesothelioma in Italy: a population-based study. Int J Cancer 2009;124(1):201-7. 
3. Rusch VW. A proposed new international TNM staging system for malignant pleural mesothelioma. From the International Mesothelioma Interest Group. Chest 1995;108(4):1122-8.

4. Britton M. The epidemiology of mesothelioma. Semin Oncol 2002;29(1):18-25.

5. Metintas M, Metintas S, Ak G, Erginel S, Alatas F, Kurt E, et al. Epidemiology of pleural mesothelioma in a population with non-occupational asbestos exposure. Respirology 2008;13(1):117-21.

6. Metintas S, Metintas M, Ucgun I, Oner U. Malignant mesothelioma due to environmental exposure to asbestos: follow-up of a Turkish cohort living in a rural area. Chest 2002;122(6):2224-9.

7. Senyiğit A, Bayram H, Babayiğit C, Topçu F, Nazaroğlu $\mathrm{H}$, Bilici A, et al. Malignant pleural mesothelioma caused by environmental exposure to asbestos in the Southeast of Turkey: CT findings in 117 patients. Respiration 2000;67(6):615-22.

8. Baris YI, Saracci R, Simonato L, Skidmore JW, Artvinli M. Malignant mesothelioma and radiological chest abnormalities in two villages in Central Turkey. An epidemiological and environmental investigation. Lancet 1981;1(8227):984-7.

9. Mandira R, Hedy LK. Malignant Pleural Mesothelioma. Chest Sept 2009;136:3.

10. Adams VI, Unni KK, Muhm JR, Jett JR, Ilstrup DM, Bernatz PE. Diffuse malignant mesothelioma of pleura. Diagnosis and survival in 92 cases. Cancer 1986;58(7):1540-51.

11. Baris YI, Sahin AA, Ozesmi M, Kerse I, Ozen E, Kolacan B, et al. An outbreak of pleural mesothelioma and chronic fibrosing pleurisy in the village of Karain/ Urgüp in Anatolia. Thorax 1978;33(2):181-92.

12. Scagliotti GV, Novello S. State of the art in mesothelioma. Annals of Oncology 2005;16(supplement 2):2405.

13. Boutin C, Schlesser M, Frenay C, Astoul P. Malignant pleural mesothelioma. Eur Respir J 1998;12(4):972-81.
14. Sterman DH, Kaiser LR, Albelda SM. Advances in the treatment of malignant pleural mesothelioma. Chest 1999;116(2):504-20.

15. Rusch VW, Venkatraman E. The importance of surgical staging in the treatment of malignant pleural mesothelioma. J Thorac Cardiovasc Surg 1996;111(4):815-26.

16. Rusch VW, Venkatraman ES. Important prognostic factors in patients with malignant pleural mesothelioma, managed surgically. Ann Thorac Surg 1999;68(5):1799-804.

17. Sugarbaker DJ, Flores RM, Jaklitsch MT, Richards WG, Strauss GM, Corson JM, et al. Resection margins, extrapleural nodal status, and cell type determine postoperative long-term survival in trimodality therapy of malignant pleural mesothelioma: results in 183 patients. J Thorac Cardiovasc Surg 1999;117(1):54-65.

18. Butchart EG. Contemporary management of malignant pleural mesothelioma. Oncologist 1999;4(6):488500.

19. Billé A, Krug LM, Woo KM, Rusch VW, Zauderer MG. Contemporary Analysis of Prognostic Factors in Patients with Unresectable Malignant Pleural Mesothelioma. J Thorac Oncol 2016;11(2):249-55.

20. Yan TD, Boyer M, Tin MM, Wong D, Kennedy C, McLean J, et al. Extrapleural pneumonectomy for malignant pleural mesothelioma: outcomes of treatment and prognostic factors. J Thorac Cardiovasc Surg 2009;138(3):619-24.

21. Flores RM, Akhurst T, Gonen M, Zakowski M, Dycoco J, Larson SM, et al. Positron emission tomography predicts survival in malignant pleural mesothelioma. J Thorac Cardiovasc Surg 2006;132(4):763-8.

22. Treasure T, Lang-Lazdunski L, Waller D, Bliss JM, Tan C, Entwisle J, et al. Extra-pleural pneumonectomy versus no extra-pleural pneumonectomy for patients with malignant pleural mesothelioma: clinical outcomes of the Mesothelioma and Radical Surgery (MARS) randomised feasibility study. Lancet Oncol 2011;12(8):763-72. 